\title{
Synthesis and Analysis of Linear Array Radiation Patterns of Tompilz Projectors
}

\author{
Fidel Souza, Eduardo Rodrigues Vale, Julio Cesar R. Dal Bello \\ ${ }^{1,2,3}$ (Telecommunications Department, School of Engineering / Federal Fluminense University, Brazil)
}

\begin{abstract}
This work aimed to synthesize, from computer simulations, radiation patterns of linear arrays of acoustic projectors of the piston type (Tompilz). This paper also has the focus to analyze the behavior of these radiation patterns when varying some parameters and characteristics of the array and radiating elements. For the array, it was considered the influence of variations related to the number of elements and the distance between them. For the elements, it was analyzed the changes in the diagrams when the product between the radius of the projector's head and the acoustic wavenumber was varied. These parameters were chosen because of his notorious influence in the shape of radiation patterns. Also, it was investigated the influence of radiation on patterns of multipath and Doppler spread, as well as the efficiency of a LMS (Least Mean Square) filter applied in the received signal. These results find application in the design of linear arrays of projectors and the development of beamforming techniques.
\end{abstract}

Keywords: array, element factor, array factor, radiation pattern, Tompilz.

\section{INTRODUCTION}

The underwater acoustic communication is a very important topic for wireless communication under the sea in the offshore oil industry, environmental monitoring, voice transmission between divers, control of autonomous underwater vehicles and mapping the ocean floor for the detection of objects and discovery of new resources.

Underwater wireless communications are usually established through the transmission of acoustic waves due to the strong attenuation suffered by the electromagnetic waves when propagating in this medium. The acoustic waves have a much lower attenuation than electromagnetic waves when propagating in submarine environment because they are mechanical waves.

Sound sources are usually named projectors and receivers are named hydrophones in the technical literature. As the antennas, projectors and hydrophones can be associated in various configurations of arrays thus allowing the creation of beams of various formats. By changing some parameters of an array of projectors or hydrophones you can change the format of the radiation pattern according to your preference. Therefore it becomes important to study the behavior of the pattern of arrays of projectors (focused in this work) and hydrophones when there are alterations in some parameters, such as the number of elements, the distance between them and some characteristics of the elements. It is also important to take into account important characteristics of the channel such as multipath and Doppler spread. For this study the simulations were performed in the software SIMULINK of MATLAB, and same situations of interest were contemplated.

This paper is structured as follows: Item II presents the mathematical considerations involved in array development; Item III gives a brief description of the simulation procedures; Item IV shows the results of the simulations developed, in the form of graphs and Item V presents the conclusions of this work.

\section{MATHEMATICAL MODELING}

In this paper it was considered the analysis of linear arrays of projectors. Therefore it is important to introduce some fundamental concepts about these type of arrays. When an array is formed by identical elements, it is possible to separate the radiation pattern of the array in two separate diagrams. The first is the diagram related to the geometry of the array, that is called array factor, and is independent of the characteristics of the elements involved. The array factor depends only on the intrinsic characteristics of the array. The second is the diagram of one element that constitutes the array that depends only on the characteristics of the element itself and is called the element factor. To obtain the complete radiation pattern it is necessary to multiply these two diagrams[1][2][3][4].

For a linear array of $N$ elements, where the currents arriving at each element are in phase, the array factor $(A F)$ is given by:

$$
A F=\sum_{n=1}^{N} \exp (j(n-1) k d \cos \theta)
$$


where $k$ is the wavenumber, $d$ is the distance between each element and $\theta$ gives the angular direction of wave propagation. The element considered in this work was the acoustic projector in the form of piston, called Tompilz. The radiation pattern of this projector was the element factor $(E F)$ considered in the simulations[5][6].

$E F=\frac{J_{1}(k a \sin \theta)}{k a \sin \theta}$

In equation (2), the term $J_{1}$ is the Bessel function of first order, $k$ is the acoustic wavenumber and $a$ is the radius of the projector's head.

\section{Simulations}

This section is dedicated to describe the programs used in this work. The first program used is responsible for generate radiation patterns of an array of two isotropic elements. The second aims to trace radiation patterns of a Tompilz projector. The third program generate diagrams of an array of two Tompilz projectors. As previously mentioned, each of these three programs are able to generate three different diagrams. A first radiation pattern is generated from an irradiated pure signal, the second diagram is generated with the main signal contaminated by Additive White Gaussian Noise (AWGN), with the addition of a reflected ray and a Doppler shift second signal, and finally a third diagram is generated from the contaminated signal after being filtered by an LMS adaptive filter.

Based on these three programs it was possible to observe the changes in the radiation patterns when parameters of interest, both of the array and the projector, were changed. It was also possible to observe the effects caused by the Doppler shift to the received signal, as well as the performance of the LMS filter.

\subsection{Array Factor Analysis}

Observations of the behavior of the array factor (AF) were performed when the distance between the elements and the number of elements in the array were changed. The parameters chosen for the test were:

- distance between the elements: $d=\lambda \in \lambda / 2$;

- number of elements: $N=2,4 \mathrm{e} 8$.

\subsection{Element Factor Analysis}

For the element factor (EF) analysis, it was varied the product between the radius of the piston's head and the acoustic wavenumber ( $\mathrm{ka}$ ) as defined in section II and the following values were considered: $\mathrm{ka}=0.92$, $3 \pi$ and $6 \pi$.

\subsection{ANALYSIS OF THE ARRAY OF TOMPILZ PROJECTORS}

As previously mentioned, the radiation pattern of an array of any element can be divided into two distinct parts: the array factor and the element factor. The array analyzed had the following settings:

- $d=\lambda$ and $\lambda / 2$;

- $\quad N=2,4$ and 8 elements;

- $k a=0,92,3 \pi$ and $6 \pi$.

\subsection{ANALYSIS OF THE INFLUENCE OF PROPAGATION CHANNEL}

In this work we also investigated the influence of the propagation channel in the received signal, in order to evaluate the distortions and the efficiency of the LMS filter. Thus, some radiation patterns with different values of the maximum Doppler shift were generated. The values considered are: $1.5 \mathrm{~Hz}, 15 \mathrm{~Hz}$ and $150 \mathrm{~Hz}$.

\section{RESUlts}

This section aims to present the results found with the simulations and an analysis about them.

\subsection{Array Factor}

As mentioned above in the analysis of the array factor, its behavior was observed when were changed the distance between each element and the number of elements in the array. The array factors with $d=\lambda$ and $N=2,4$ e 8 elements are shown in Fig.1 and produces radiation patterns with many null points (Nnull) that obey the following empirical equation:

$N_{\text {null }}=2 N-2 \quad N=2,4,8 \ldots$

The array factors with $d=\lambda / 2$ e $N=2,4$ and 8 elements are shown in Fig. 2. Note that the number of null points are:

$N_{\text {null }}=N \quad N=2,4,8 \ldots$ 

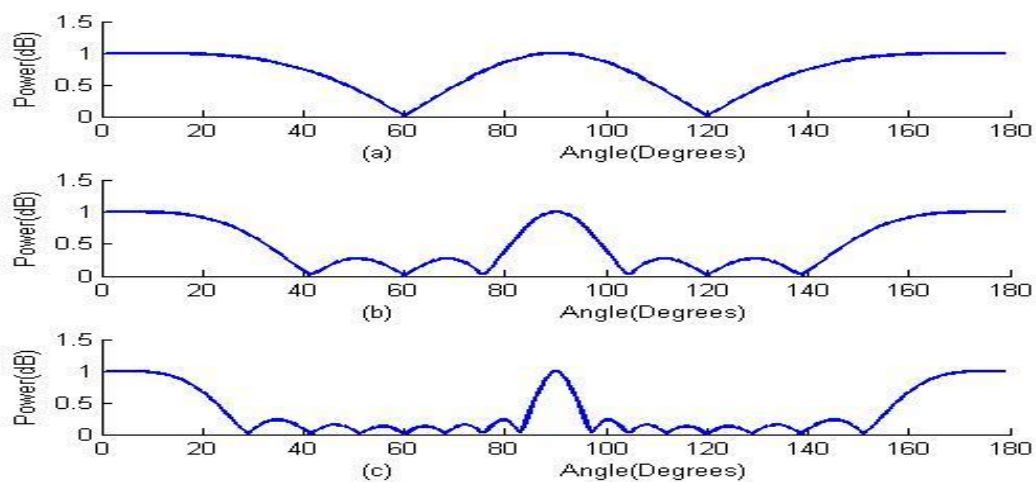

Figure 1: Array Factor - Radiation Patterns with $d=\lambda$ and (a) $N=2$ (b) $N=4$. (c) $N=8$
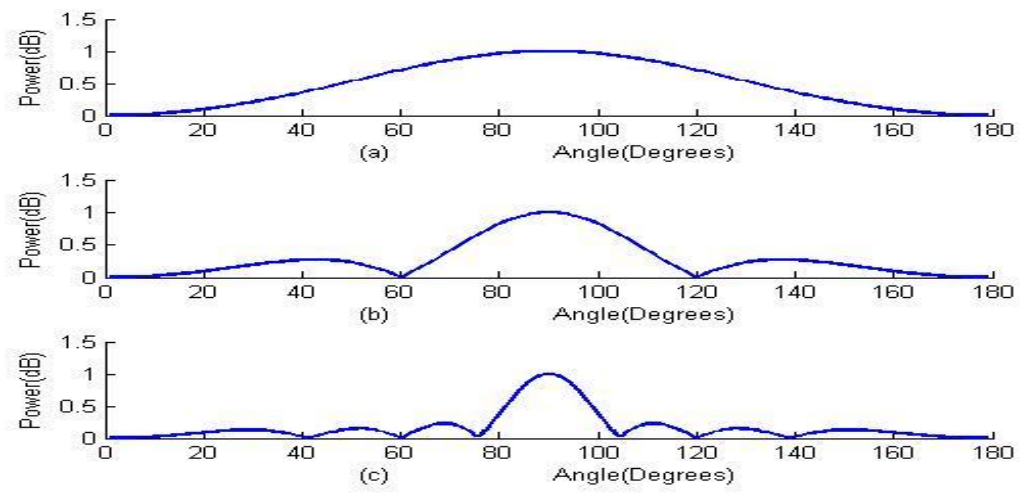

Figure 2: Array Factor - Radiation Patterns with $d=\lambda / 2$ with (a) $N=2$, (b) $N=4$ and (c) $N=8$.

\subsection{Element Factor}

The Fig. 3 shows the element factors diagrams that were produced with $k a=0.92,3 \pi$ e $6 \pi$. When the value of ka grows, it raises the number of null points of the radiation pattern and increases the projector's directivity. As ka is the product of the acoustic wavenumber (k) and the piston's head radius (a), we have:

$k a=\frac{2 \pi a}{\lambda}=\frac{2 \pi a}{c} f$

where $f$ is the frequency and $c$ is the sound velocity.

The equation (5) indicates that the shape of the element factor diagram is dependent of the projector operating frequency. It is very important to observe that the radiation pattern with ka $=0.92$ is a smooth curve without null points and with a little peak at $\theta=\pi / 2$. In other words, the Tompilz transducer with this configuration radiates power almost uniformly over $\theta$.
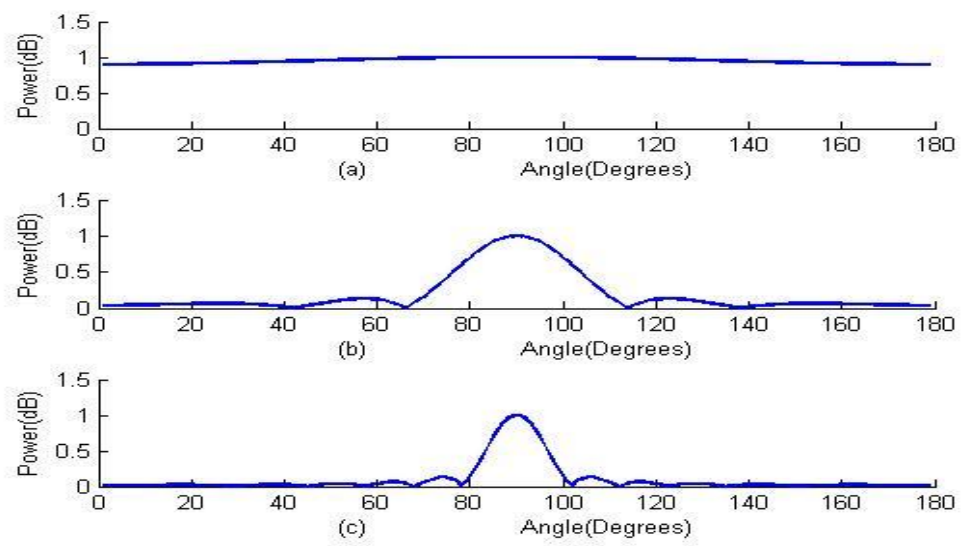

Figure 3: Element Factor - Radiation Patterns with (a) $k a=0.92$, (b) $k a=3 \pi$ and (c) $k a=6 \pi$. 


\subsection{Arrays Of Tompilz Projectors}

As discussed above, the radiation pattern of an array of Tompilz projectors is formed by the product of array factor and element factor and radiation patterns of different formats were obtained using different configurations. The Fig. 4 shows the radiation patterns of the arrays of projectors with $d=\lambda$, $k a=0.92$ and $N$ $=2,4$ and 8 elements. It is noticed that they do not differ very much from the array factors diagrams brought by Fig. 1, because the element factor with $\mathrm{ka}=0.92$ is almost a constant curve. An interesting fact is that with this value of ka, the shape of the radiation pattern depends almost exclusively of the form of the array factor. The Figs. 5 and 6 show the radiation patterns when $\mathrm{ka}=3 \pi$ and $6 \pi$. The results are extremely directive diagrams, with very narrow beams and with high incidence of null points.
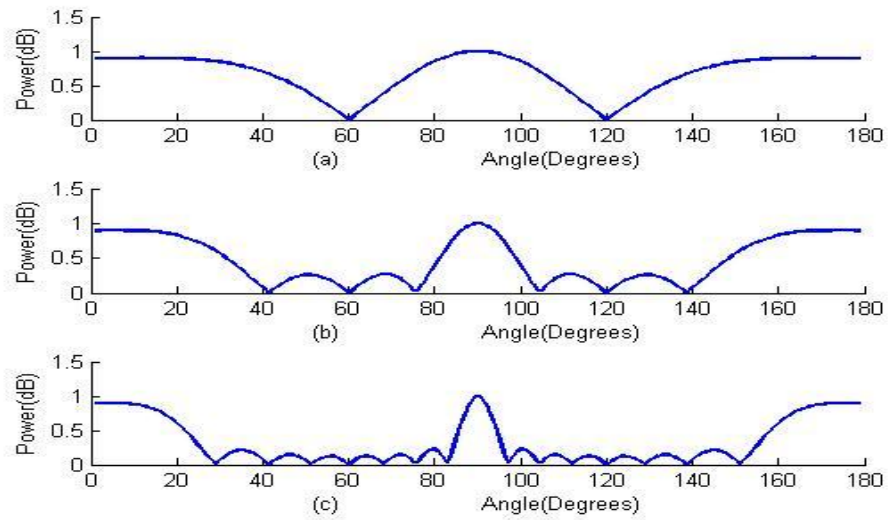

Figure 4: Radiation Patterns of an Array of Tompilz Projectors with: $d=\lambda, k a=0.92$ and N=2,4,8 elements.
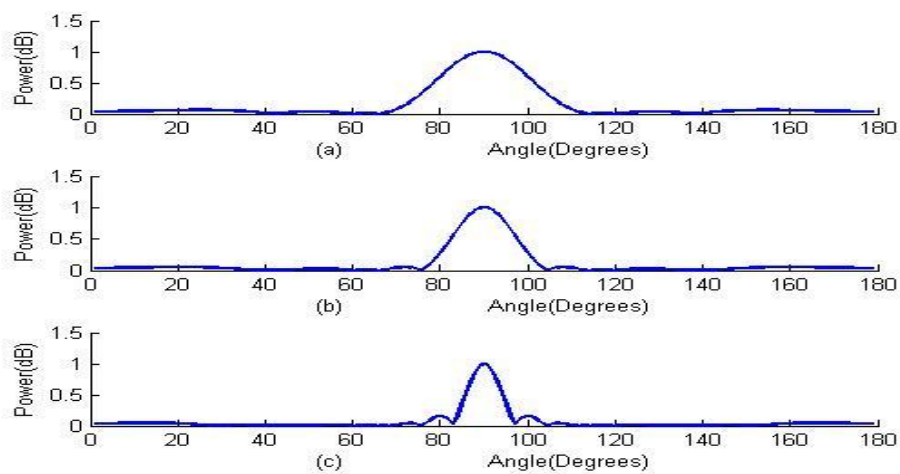

Figure 5: Radiation Patterns of an Array of Tompilz Projectors with: $d=\lambda, k a=3 \pi$ and $\mathrm{N}=2,4,8$ elements
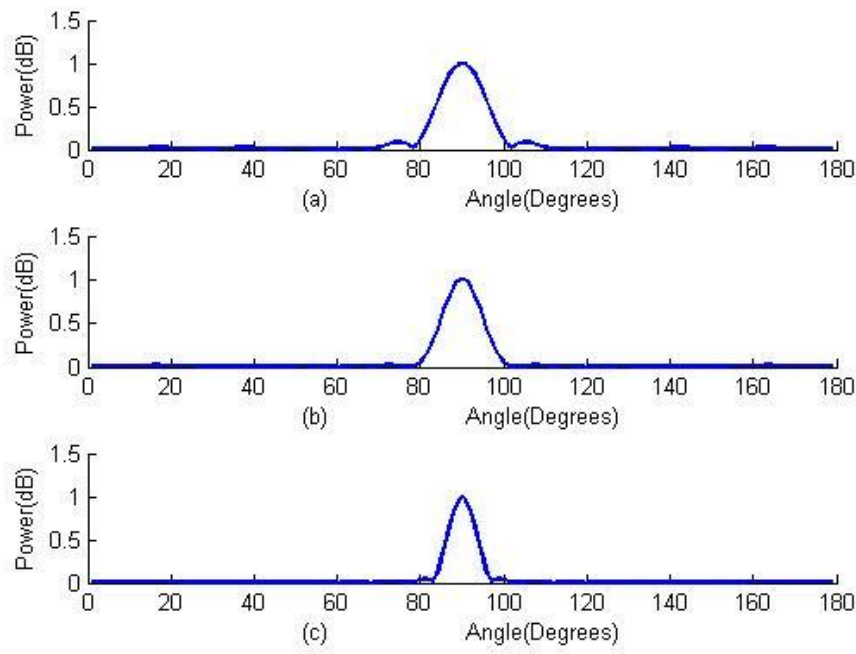

Figure 6: Radiation Patterns of an Array of Tompilz Projectors with: $d=\lambda, k a=6 \pi$ and $\mathrm{N}=2,4,8$ elements 


\subsection{Doppler Spread And Multipath}

As mentioned earlier, in this study it was also analyzed the influence of some parameters, of the propagation channel on the received signal. For this purpose, radiation patterns of an array of Tompilz projectors was generated, using a signal formed by a direct ray and a reflected one, both subject to Doppler shift.
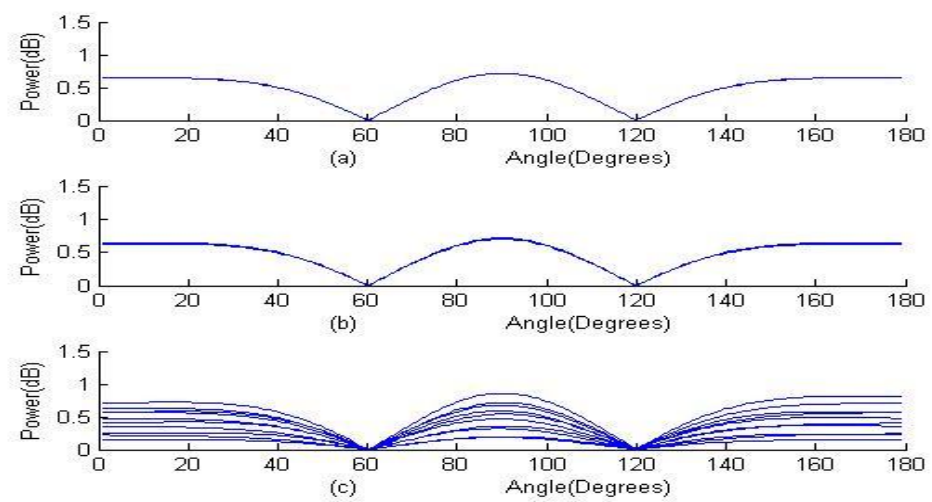

Figure 7: Maximum Doppler Shift: (a) $1.5 \mathrm{~Hz}$, (b) $15 \mathrm{~Hz}$ e (c) $150 \mathrm{~Hz}$.

The values adopted for the maximum Doppler shift were 1.5, 15 and $150 \mathrm{~Hz}$. The results are shown in Fig 7, which gives a superposition of the radiation patterns of an array of projectors with $\mathrm{d}=\lambda, \mathrm{ka}=0.92$ and $\mathrm{N}=2$, at different instants of time.

The Fig. 7 shows that the Doppler shift with multipath effects bring an oscillation of the power level of the received signal, and this oscillation appears larger when it is increased the maximum Doppler shift parameter. This is expected since the signal is a sum of two lagged rays, which can result in constructive or destructive interference, depending on the phase difference between the two rays. The results of this analysis also indicated that the position of the null points remain more or less fixed, as can be seen in Figure 7, a phenomenon in principle unexpected. One possible explanation for this phenomenon can be found by analyzing the following equation:

$\theta_{\text {null }}=\cos ^{-1}\left(\frac{1}{k d}\left( \pm \frac{2 n \pi}{N}-\delta\right)\right), n=1,2,3 \ldots$

that provides angular null positions of the array factor. Considering the Doppler effect and changes in the density of water along the propagation path, for both the direct ray and the reflected ray, it can be considered that:

$\lambda^{\prime}=\lambda+\Delta \lambda$

(7)

then:

$k^{\prime}=\frac{2 \pi}{\lambda^{\prime}}=\frac{2 \pi}{\lambda+\Delta \lambda}$

If the distance between the elements is taken as a linear function of the original wavelength, in other words:

$d=\alpha \lambda$

(9)

then (6) becomes:

$\theta_{\text {null }}=\cos ^{-1}\left(\frac{1}{\frac{2 \pi}{\lambda+\Delta \lambda} \alpha \lambda}\left( \pm \frac{2 n \pi}{N}-\delta\right)\right), n=1,2,3 \ldots$

(10)

Considering that $\delta=0 \mathrm{e} \lambda>>\Delta \lambda$, (6) reduces to:

$\theta_{\text {null }}=\cos ^{-1}\left(\frac{n}{\alpha N}\right), n=1,2,3 \ldots$

Therefore, if the wavelength variation can be considered small compared to the original wavelength, the position of the null points does not change significantly. 


\subsection{LMS FILTER}

In this study it was also evaluated the effectiveness of the utilization of an adaptive filter with four coefficients using the least mean square algorithm (LMS). The Fig. 8 shows the results and can be noticed that the filter is effective in recovering the original signal characteristics, but when Doopler shift becomes very large, the efficiency of the filter becomes smaller.
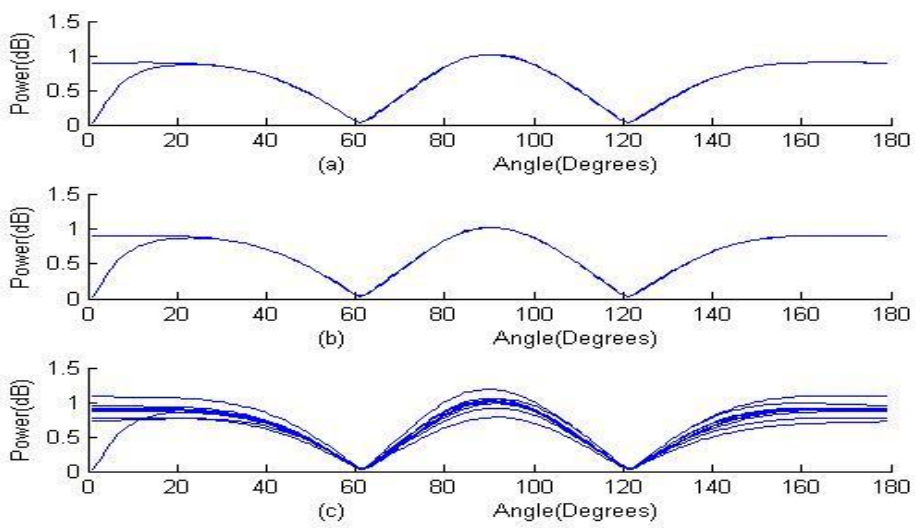

Figure 8: Filtered Signal with Maximum Doppler shift: (a) $1.5 \mathrm{~Hz}$, (b) $15 \mathrm{~Hz}$ and (c) $150 \mathrm{~Hz}$.

\section{CONCLUSIONS}

This study aimed to analyze, using simulations performed in the software SIMULINK of MATLAB, the radiation patterns of arrays formed with Tompilz transducers. Firstly, it was analyzed the diagrams referring to the array factor and, afterwords, the diagrams referring to the element factor were analyzed. Finally, the product between the array factor diagram and the element factor diagram, representing the radiation patterns of arrays of Tompilz transducers, were discussed.

It was also analyzed the changes of the array factor when the number of elements $\mathrm{N}=2,4$ and 8 varies with the distance between them given by $d=\lambda$. In this analysis it was concluded that when the number of elements grows, the number of null points raises too, obeying the following equation: $N u l_{d=\lambda}=2 N-2$. The behavior of the array factor for $N=2,4$ and 8 and for the distance equal to $d=\lambda / 2$ were also analyzed. In these diagrams the number of null points is less than the previous case, obeying the following equation: $N u l_{d=\lambda / 2}=N$. With the distance between the elements equal to $\lambda / 2$, the diagrams are less directive that when the distance is equal to $\lambda$.

The analysis of the behavior of the element factor for Tompilz transducers with different values of $k a$ were developed. The values chosen were: $k a=0.92,3 \pi$ e $6 \pi$. With this analysis, it was concluded that when the value of $k a$ increases, the number of null points at the element factor also increases.

Observations were also made about the radiation patterns of the array with Tompilz transducers. It were generated the analysis of diagrams with $N=2,4$ e $8, \mathrm{ka}=0.92,3 \pi \mathrm{e} 6 \pi$, for $\mathrm{d}=\lambda$. From these analyses it was concluded that for $\mathrm{ka}=0.92$ and $N=2,4$ e 8 the diagrams are very close to the array factors diagrams related to this configuration, and also keeping the number of null points. This is due to the fact that the element factor of the transducer with $\mathrm{ka}=0.92$ is a smooth curve without null points, and almost constant, with a little peak in $\theta=\pi / 2$. It was observed that the diagrams formed with $N=2,4,8$ and $\mathrm{ka}=3 \pi$, are very different for arrays with equal values for $N$. The radiation patterns with $\mathrm{ka}=6 \pi$ have a high directivity and a large number of null points.

In this paper we investigated the behavior of radiation patterns when the parameter maximum Doppler shift is varied. For this analysis, the values chosen were: $1.5 \mathrm{~Hz}, 15 \mathrm{~Hz}$ and $150 \mathrm{~Hz}$. The array had $N=2, \mathrm{~d}=\lambda$, and $\mathrm{ka}=0.92$. In these analyses it was observed that the Doppler shift brought a fluctuation in the average signal power, and the higher the deviation the greater the buoyancy. We also noticed that the positions of null points at the diagrams were little changed. This is because the wavelength variation is small compared to the wavelength itself. According to the knowledge of the authors, there is no specific technical literature that deals with this subject.

Finally it has been seen that an adaptive filter can improve the signal reception, but if the Doppler shift is very large, the filter becomes ineffective.

Currently, the authors are working on experimental proof of the simulations results, by performing tests in an environment of underwater acoustics, with arrays of Tompilz projectors. 


\section{REFERENCES}

[1] C. A. Balanis, Antenna theory: analysis and design (Hoboken, NJ: Wiley Interscience, 2005).

[2] W. L. Stutzman e G. A. Thiele, Antenna theory and design ( Hoboken: Wiley, 2013).

[3] F. B. Gross, Smart antennas for wireless communications with MATLAB ( New York; London: McGraw-Hill; McGraw-Hill [distributor], 2005).

[4] J. D. Kraus e R. J. Marhefka, Antennas for all applications ( New York: McGraw-Hill, 2002).

[5] C. H. Sherman e J. L. Butler, Transducers and arrays for underwater sound ( New York; London: Springer, 2011).

[6] R. P. Hodges, Underwater acoustics analysis, design, and performance of sonar (Hoboken, NJ: Wiley, 2010). 